\title{
Comparison of two commercial molecular tests for the detection of Clostridium difficile in the routine diagnostic laboratory
}

\section{Correspondence \\ Maja Rupnik \\ maja.rupnik@zzv-mb.si}

Received 12 January 2011

Accepted 25 February 2011

\author{
Valerija Zidarič, ${ }^{1}$ Bozena Kotnik Kevorkijan, ${ }^{2}$ Nadja Oresic, ${ }^{1}$ \\ Sandra Janezic ${ }^{1}$ and Maja Rupnik ${ }^{1,3,4}$ \\ ${ }^{1}$ Institute of Public Health Maribor, Maribor, Slovenia \\ ${ }^{2}$ Department of Infectious Diseases, University Medical Center Maribor, Ljubljanska ulica 5, 2000 \\ Maribor, Slovenia \\ ${ }^{3}$ Faculty of Medicine, University of Maribor, Maribor, Slovenia \\ ${ }^{4}$ Centre of Excellence for Integrated Approaches in Chemistry and Biology of Proteins (CIPKEBIP), \\ Ljubljana, Slovenia
}

Two commercial real-time PCR assays for the detection of Clostridium difficile, BD GeneOhm Cdiff assay (BD Diagnostics) and Xpert C. difficile assay (Cepheid), were compared to each other and to toxigenic culture, which was used as a gold standard, on a set of 194 clinical stools submitted for routine diagnostic analysis. Of 28 (14.4\%) toxigenic culture positive samples 23 were positive with both assays, the BD and the Cepheid real-time PCR assays, 4 were positive only by Cepheid Xpert $C$. difficile assay and 1 sample was negative by both PCR assays, resulting in sensitivity, specificity, positive predictive value and negative predictive value of $82.1,98.2,88.5$ and 97.0\%, respectively, for the BD GeneOhm Cdiff assay, and 96.4, 97.3, 87.1 and 99.3\%, respectively, for the Cepheid Xpert C. difficile assay. Altogether 26 out of 194 (13.4\%) samples were reported invalid by Cepheid. Toxigenic C. difficile positive samples contained 15 different PCR ribotypes distributed into toxinotype 0 and 2 different variant toxinotypes (III, IV). Clinical data were available for 24 out of $28(85.7 \%)$ toxigenic C. difficile positive patients and $18(75.0 \%)$ of them were diagnosed with diarrhoea, while others had other symptoms or risk factors related to possible C. difficile infection (antibiotics, bloody stool, peritonitis, Crohn's disease).

\section{INTRODUCTION}

Clostridium difficile infection (CDI) is one of the most important causes of nosocomial diarrhoea and is also becoming important in the community and in populations with low or no risk factors (Rupnik et al., 2009). CDI can result in asymptomatic colonization or can result in clinical symptoms ranging from mild diarrhoea to severe colitis, leading to complications such as toxic megacolon or sepsis. There are a variety of commercially available diagnostic tests for the diagnosis of $C$. difficile, differing in turnaround time, sensitivity, specificity, costs, workload and availability (Crobach et al., 2009). ELISAs are the most commonly employed tests in C. difficile diagnosis. They are rapid and inexpensive but lack sensitivity (Planche et al., 2008).

Especially during outbreaks and during the spread of strains with increased virulence it is important that the laboratory diagnosis is rapid and sensitive to ensure

Abbreviations: $\mathrm{CDI}$, Clostridium difficile infection; $\mathrm{Cl}$, confidence interval. appropriate therapy and quick implementation of hospital hygiene measures. Therefore several groups have reported in-house real-time PCR assays. All had very good sensitivity, specificity and short turnaround times but special procedures for elimination of PCR inhibitors during DNA extraction were needed (Alonso et al., 1999; Bélanger et al., 2003; Van den Berg et al., 2005; Eastwood et al., 2009; Wroblewski et al., 2009). Currently several realtime PCR assays that detect toxigenic $C$. difficile directly from stool are commercially available. Published reports cover only three of them: BD GeneOhm Cdiff assay (BD Diagnostics), ProGastro Cd assay (Prodesse) and Cepheid Xpert $C$. difficile, which detect the toxin $\mathrm{B}$ gene $(t c d \mathrm{~B})$ (Barbut et al., 2009; Huang et al., 2009; Stamper et al., 2009; Terhes et al., 2009; Babady et al., 2010; Doing et al., 2010; Knetsch et al., 2011; Kvach et al., 2010; NovakWeekley et al., 2010; Swindells et al., 2010; Tenover et al., 2010). Cepheid Xpert C. difficile also gives information about the binary toxin gene and $117 \mathrm{bp} t c d C$ deletion, both features of the NAP1/BI/027 strain, a causative agent for epidemics of CDI in countries worldwide. A fourth 
commercially available assay is based on the ligase chain reaction (illumigene $C$. difficile; Meridian) and detects the tcdA gene (Norén et al., 2011).

The aim of this study was to compare two commercial molecular tests to the toxigenic culture method performed as a part of routine diagnostics. In addition, detection of $C$. difficile was correlated with clinical data and with the genotype (PCR ribotype and toxinotype) of the strain.

\section{METHODS}

Stool specimens. Samples from hospitalized patients and patients from other institutions ( $<5 \%$ of all samples; sent by general practitioners and long-term care facilities) submitted to the Institute of Public Health, Maribor, for routine C. difficile testing between November 2008 and April 2009 were included in the study. Samples were either liquid $(62.4 \%)$, semi-solid $(12.4 \%)$ or solid $(14.4 \%)$, while for $10.8 \%$ of samples the consistency was not determined. Samples with sufficient stool available were tested with two commercial real-time PCRs within 2 to 3 days after collection, and were stored during this time at $4{ }^{\circ} \mathrm{C}$. If testing in this time frame was not possible samples were stored at $-25^{\circ} \mathrm{C}$ until use.

Isolation of C. difficile. During the routine isolation of C. difficile, stool samples were subjected to alcohol shock and cultured on commercially available CLO agar plates (bioMérieux). Initial identification was based on the morphology of the colonies and typical odour, and was confirmed by the VITEK system (bioMérieux). The VIDAS C. difficile toxin A and B assay (bioMérieux) was used for the detection of toxins directly from stool samples or from multiple colonies from positive primary cultures if the toxin result from the sample was negative.

A sample with isolated $C$. difficile that produces toxin A and/or B according to a positive toxin test was defined as a positive routine toxigenic culture. If the isolated strain did not produce toxins, the sample was defined as a routine nontoxigenic culture.

In the case of a negative routine culture result and a positive result by at least one molecular test, enrichment from the stool sample was performed in CCFB (cycloserine-cefoxitine-fructose broth) (which has the same ingredients as Oxoid C. difficile agar minus the agar), supplemented with D-cycloserine and cefoxitine (C. difficile selective supplement; Oxoid) for 5 days in anaerobic jars at $37^{\circ} \mathrm{C}$. After incubation, an equal amount of ethanol was added to $500 \mu \mathrm{l}$ enrichment culture, this was incubated for $30 \mathrm{~min}$ at room temperature and centrifuged at $10000 \mathrm{~g}$ for $5 \mathrm{~min}$. The entire pellet was inoculated onto CLO agar plates (bioMérieux). One or more recovered $C$. difficile colonies were subcultured on blood agar plates.

Characterization of C. difficile isolates. Toxinotyping and PCR ribotyping were performed on strains subcultured for 24-48 h on Columbia blood agar as described by Rupnik (2008) and Bidet et al. (1999). Binary toxin genes were detected by PCR, as described by Stubbs et al. (2000). The PaLoc-negative genotype was confirmed by PCR using Lok1/Lok3 primers (Braun et al., 1996). PCR ribotype profiles were analysed by BioNumerics software and PCR ribotypes were designated either with standard Cardiff nomenclature $(001, \ldots)$ if the profile corresponded to one of 25 reference PCR ribotype strains, including the current most frequent PCR ribotypes (including among others $001,002,014,015,023,027,078,106,126, \ldots)$, or with internal nomenclature (SLO 001, ...).

Molecular tests. Both molecular tests were performed directly on stool samples. Molecular testing and routine culture/toxin testing were each carried out independently by different members of the laboratory. If commercial PCR failed to detect $t c d \mathrm{~B}$ directly from toxigenic culture positive stool, isolates were also analysed by the same commercial PCR to test the detection of the isolated strains by these assays.

BD GeneOhm Cdiff assay. The BD GeneOhm Cdiff assay was performed according to the manufacturer's protocol. Lysis of the specimen (faeces or 1-5 C. difficile colonies cultured for $24 \mathrm{~h}$ on blood agar plates) was performed by vortexing the sample with glass beads, heating and cooling. The lysate was added to a SmartCycler tube containing reconstituted master mix. Every PCR run included a PCR positive control (reconstituted DNA from the manufacturer's kit). Uninoculated sample buffer served as a negative control. Every sample assay also included an internal control. The specific reaction tubes were placed in the SmartCycler I-CORE module (Cepheid) and run using Cepheid SmartCycler software with the BD GeneOhm Cdiff amplification protocol. The interpretation of the assay results was reported as: 'negative' where no $t c d B$ gene was detected, 'positive' where $t c d B$ gene was detected, 'unresolved' in case of inhibition of internal control or reagent failure, 'invalid assay run' where one of the PCR controls (positive or negative) failed and 'not determined' when there was an I-CORE module malfunction.

In the case of a reported 'invalid' or 'unresolved' assay run the assay should, according to the manufacturer's protocol, be repeated with the same lysate (crude DNA). No such results were obtained during this study.

Cepheid Xpert C. difficile assay. The Cepheid Xpert C. difficile assay was performed according to the manufacturer's protocol. A sterile Copan swab was dipped into the stool specimen and material was resuspended in the sample buffer and transferred to the single-use disposable cartridge. The amplification and detection was run in the GeneXpert Dx module. Every run included the SPC (sample processing control), the control for adequate processing of the target bacteria and to monitor inhibition of PCR, and the PCC (probe check control), the control that verified reagent rehydration, PCR tube filling in the cartridge, probe integrity and dye stability. Results were automatically interpreted by the software as: ' $C$. difficile positive', ' $C$. difficile 027 NAP1 presumptive positive', ' $C$. difficile negative', 'invalid', 'error' or 'no result'. Also, the Cepheid assay enabled the viewing of amplification curves and hence interpretation by the technician. In the case of 'invalid', 'error' or 'no result', the reported test was repeated. Retesting was performed using a new swab sample taken from the original stool sample.

\section{RESULTS}

\section{Results of both molecular tests compared to routine toxigenic culture}

A total of 194 samples were analysed by both molecular tests (BD and Cepheid) and the results compared to toxigenic culture. Of all 194 tested samples, 28 (14.4\%) were toxigenic culture positive in the routine testing (without enrichment) (Table 1). Initially, 23 (11.9\%) samples were positive by all three assays and 141 (72.7\%) were negative by all three assays. Of the 28 routine toxigenic culture positive samples, 4 were positive only by Cepheid test and not by BD test.

At initial testing 26 (13.4\%) invalid (reported as invalid or error) results were obtained by the Cepheid assay. Of them 
Table 1. Comparison of two molecular tests to toxigenic culture obtained in the routine laboratory

\begin{tabular}{|c|c|c|c|c|c|c|}
\hline \multirow[b]{2}{*}{ Toxigenic culture } & \multicolumn{3}{|c|}{ Xpert C. difficile (Cepheid) } & \multicolumn{3}{|c|}{ GeneOhm Cdiff (BD) } \\
\hline & Positive & Negative & $\begin{array}{l}\text { Total no. of } \\
\text { samples }\end{array}$ & Positive & Negative & $\begin{array}{l}\text { Total no. of } \\
\text { samples }\end{array}$ \\
\hline Positive & 27 & 1 & 28 & 23 & 5 & 28 \\
\hline Negative & 4 & 146 & 150 & 3 & 163 & 166 \\
\hline $\begin{array}{l}\text { Total no. of } \\
\text { samples }\end{array}$ & 31 & 147 & $178^{\star}$ & 26 & 168 & 194 \\
\hline
\end{tabular}

${ }^{\star}$ Samples that gave an invalid result by molecular methods were repeated. If the result was positive or negative, the sample was included in the analysis; if the result was invalid again the sample was excluded from the analysis ( $n=16$ samples).

two were routine toxigenic culture positive and one was positive after $C$. difficile enrichment (all three were BD positive). After repeated testing Cepheid resolved the result in 10 out of 26 samples. The remaining 16 'invalid' samples were excluded from further analysis (Table 1). None of the samples was invalid by BD assay.

Compared to the routine toxigenic culture, and after exclusion of invalid results, the sensitivity ( $95 \%$ confidence interval) $(95 \% \mathrm{CI})$, specificity $(95 \% \mathrm{CI})$, positive predictive value $(95 \% \mathrm{CI})$ and negative predictive value $(95 \%$ $\mathrm{CI})$ for the BD GeneOhm assay $(n=194)$ were: $82.1 \%$ (67.9-96.3), 98.2\% (96.2-100.0), $88.5 \%(76.2-100.0)$ and $97.0 \%$ (94.4-99.6), respectively, and for the Cepheid Xpert C. difficile assay $(n=178) 96.4 \%(89.5-100.0), 97.3 \%$ (94.7-99.9), 87.1\% (75.3-98.9) and 99.3\% (98.0-100.0), respectively (Table 1 ).

In a total of 12 samples, the results of one or both molecular tests were not in agreement with routine toxigenic culture, but all discrepant results were clarified after repeated testing or after enrichment (Table 2). In cases where retesting of the false negatives with $\mathrm{BD}$ and/or Cepheid assay resulted in a negative outcome, isolated strains from that sample were also tested with the same molecular test and were all recognized. The outcome for six out of seven routine toxigenic culture negative samples was a positive result with at least one of the molecular tests (Table 2) and one was indicated to be positive only by Cepheid assay.

\section{Detection of variant and nontoxigenic strains by molecular tests}

C. difficile was cultured from 38 out of 194 stool samples. In addition to 28 toxigenic strains, 5 nontoxigenic strains were isolated by routine culture (SLO 010, SLO 028, SLO 030, SLO 055, SLO 057), and an additional 5 samples yielded toxigenic $C$. difficile after enrichment. Neither of the commercial molecular tests reported the $t c d B$ marker in five stool samples that yielded nontoxigenic strains.

From 38 samples a total of 39 strains were obtained. The majority $(94.9 \%)$ of isolated toxigenic strains belonged to toxinotype $0(014 / 020$, SLO 009, SLO 022, SLO 026, 002,
SLO 011, SLO 050, SLO 007, 029, SLO 071, SLO 016, SLO 068, SLO 074). Only two strains were variant toxinotypes: toxinotype IV (binary toxin positive; PCR ribotype 023) and toxinotype III (binary toxin negative; PCR ribotype SLO 049). Both molecular tests detected the $t c d B$ gene in toxinotype 0 , toxinotype III and toxinotype IV strains. In the latter strain (IV/023), binary toxin gene was also correctly identified by Cepheid test. Out of 15 different PCR ribotypes, 7 were not always detected by one or both molecular tests (Table 2). None of the samples contained all three genetic markers $(t c d B$, binary toxin and $t c d C$ deletion) and no PCR ribotype 027 was recovered from any of the culture positive samples.

\section{Clinical data and positive samples}

Clinical data were available for 28 out of $38(73.7 \%) C$. difficile positive samples, including toxigenic and nontoxigenic strains. C. difficile was detected at least once in 24 out of $170(14.1 \%)$ sampled patients. During the study period 14 patients were sampled only once, 4 patients were sampled twice, and for a small number of patients three samples $(n=3)$, four samples $(n=2)$ and five samples $(n=1)$ were taken. For most of patients with multiple samples the time frame between the first and last sample was from 1 to 3 months. Selected patients with more than one sample are shown in Table 3.

Clinical signs (diarrhoea, colitis or pseudomembranous colitis) correlated with toxigenic culture in 18 out of 24 $(75.0 \%)$ toxigenic culture positive samples, where clinical data were available. Other patients did not have signs of diarrhoea at the time of sampling but were tested for $C$. difficile due to other reasons, like severe abdominal pain, previous antibiotic treatment, an episode of inflammatory bowel disease and vomiting.

\section{DISCUSSION}

The aim of our study was to compare the detection of $C$. difficile in samples from patients suspected of having CDI with two commercial molecular $C$. difficile diagnostic assays. Valid results of both tests were in congruence with 
Table 2. Resolution of discrepant results

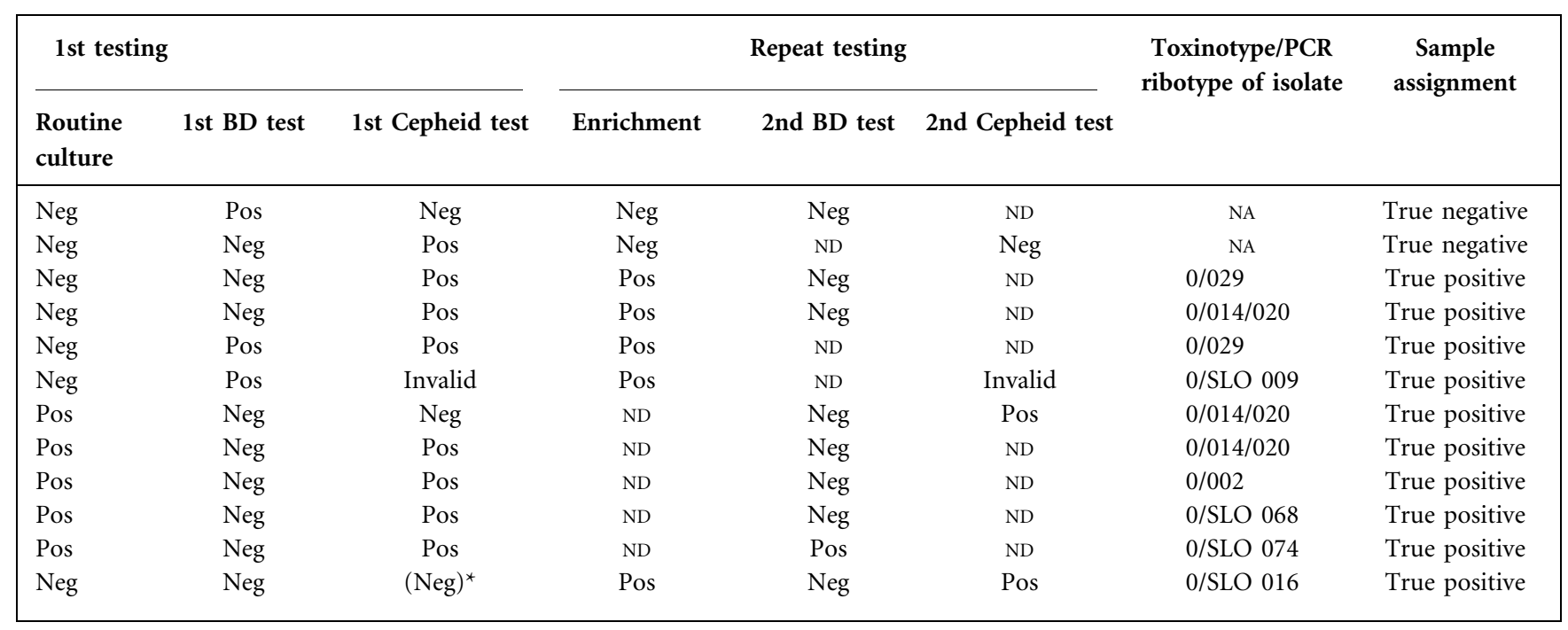

NA, Not applicable; ND, not done; neg, negative; pos, positive.

${ }^{\star}$ Cepheid test reported as negative, but the amplification curve was rising after the cut-off and hence the test was repeated.

toxigenic culture results obtained in the routine laboratory. In some cases the molecular diagnostic test was even more sensitive than the usual routine culture, and C. difficile was isolated from these samples only after enrichment. Most of the $28 \mathrm{C}$. difficile toxigenic culture positive cases with obtained clinical data were presented as with either diarrhoea or some other gastrointestinal symptoms.
It was reported that the PCR ribotype can affect the sensitivity of some enzymic diagnostic tests and that, for example, PCR ribotype 027 is more likely to be recognized by immunological tests than some other prevalent PCR ribotypes (e.g. ribotype 014) (Tenover et al., 2010). In our study 9 out of $33(27.3 \%)$ toxigenic culture positive samples (routine and enrichment cultures) resulted in false

Table 3. Collected data and testing results of selected patients sampled more than once

\begin{tabular}{|c|c|c|c|c|c|c|c|c|c|c|}
\hline Patient & $\begin{array}{l}\text { Routine } \\
\text { culture }\end{array}$ & Enrichment & $\begin{array}{c}\text { Cepheid } \\
\text { test }\end{array}$ & $\begin{array}{l}\text { Cepheid } \\
\text { repeat test }\end{array}$ & $\begin{array}{l}\text { BD } \\
\text { test }\end{array}$ & $\begin{array}{c}\text { BD repeat } \\
\text { test }\end{array}$ & $\begin{array}{l}\text { Sampling } \\
\text { date }\end{array}$ & $\begin{array}{c}\text { Toxinotype/PCR } \\
\text { ribotype }\end{array}$ & Symptoms & $\begin{array}{l}\text { Antibiotic } \\
\text { treatment }\end{array}$ \\
\hline \multirow[t]{3}{*}{ Patient 1} & tox & $\mathrm{ND}$ & + & ND & + & ND & 28.11 .2008 & 0/SLO 009 & $\mathrm{~d} / \mathrm{col}$ & No \\
\hline & tox & $\mathrm{ND}$ & Invalid & + & + & ND & 15.01 .2009 & 0/SLO 009 & $\mathrm{~d}$ & No data \\
\hline & - & tox & Invalid & Invalid & + & ND & 25.02.2009 & 0/SLO 009 & $\mathrm{~d}$ & No \\
\hline \multirow[t]{5}{*}{ Patient 2} & - & $\mathrm{ND}$ & - & $\mathrm{ND}$ & - & ND & 12.01 .2009 & Neg & No data & No data \\
\hline & - & ND & - & $\mathrm{ND}$ & - & ND & 15.01 .2009 & $\mathrm{Neg}$ & No data & No data \\
\hline & - & tox & + & $\mathrm{ND}$ & - & - & 12.02 .2009 & $0 / 029$ & No data & No data \\
\hline & tox & ND & + & ND & + & ND & 17.02.2009 & $0 / 029$ & $\mathrm{a}$ & Yes \\
\hline & tox & ND & + & ND & + & ND & 08.03.2009 & $0 / 029$ & $\mathrm{~d}$ & Yes \\
\hline \multirow[t]{4}{*}{ Patient 3} & tox & ND & + & $\mathrm{ND}$ & + & ND & 01.12 .2008 & $0 / 014 / 020$ & $\mathrm{a}$ & Yes \\
\hline & tox & ND & + & $\mathrm{ND}$ & + & $\mathrm{ND}$ & 10.12 .2008 & 0/SLO 022 & $\mathrm{~d}$ & Yes \\
\hline & tox & ND & + & ND & + & ND & 07.01 .2009 & $0 / 014 / 020$ & $\mathrm{~d} / \mathrm{pmc}$ & No \\
\hline & - & ND & + & - & - & ND & 26.01 .2009 & Neg & a & Yes \\
\hline \multirow[t]{4}{*}{ Patient 4} & tox & ND & + & ND & + & ND & 31.12 .2008 & 0/SLO 011 & $\mathrm{~d}$ & Yes \\
\hline & tox & ND & + & ND & + & ND & 19.01.2009 & 0/SLO 011 & No data & No data \\
\hline & tox & $\mathrm{ND}$ & + & $\mathrm{ND}$ & - & - & 30.01 .2009 & 0/SLO 068 & a & No \\
\hline & - & tox & + & $\mathrm{ND}$ & + & ND & 24.02 .2009 & $0 / 029$ & $\mathrm{~d}$ & Yes \\
\hline \multirow[t]{3}{*}{ Patient 5} & nontox & $\mathrm{ND}$ & - & $\mathrm{ND}$ & - & ND & 19.01.2009 & tox-/SLO 010 & No data & No \\
\hline & nontox & ND & - & $\mathrm{ND}$ & - & ND & 22.01 .2009 & tox-/SLO 055 & $\mathrm{a}$ & No \\
\hline & - & tox & - & + & - & - & 07.02.2009 & 0/SLO 016 & $\mathrm{~d}$ & Yes \\
\hline
\end{tabular}

a, Asymptomatic; col, colitis; d, diarrhoea; ND, not done; neg, no isolated strains; nontox, nontoxigenic C. difficile; pmc, pseudomembranous colitis; tox, toxigenic C. difficile. 
negatives by at least one of the two molecular assays. These nine isolates were grouped into seven different PCR ribotypes and no association was seen between falsenegative results and specific genotypes isolated from the samples.

Although both molecular assays are validated and suggested for use on liquid or unformed stools, the diagnostic laboratory would often receive formed stool samples for testing. These were mainly from patients with symptoms other than diarrhoea, such as abdominal pain, fever or a previous $C$. difficile episode. In our study only $62.4 \%$ samples were unformed, and the results suggest that consistency did not affect sensitivity and was not associated with invalid results.

The BD test did not give any invalid results. A low percentage of invalid results was reported by Stamper et al. (2009) and Terhes et al. (2009). In our study the Cepheid test had a higher proportion of invalid results and they were rarely resolved after repeating the test. This was not reported previously. However, the Cepheid test allows viewing of the amplification curves. In our study, one sample had all tests reported as negative but because the Cepheid assay showed a very late increase of the amplification curve we repeated the test, as well as performing an enrichment culture, and both resulted in a positive outcome. At this point, the patient was also symptomatic (Table 2; Table 3, patient 5).

Both molecular assays were found to be useful for routine diagnosis of $C$. difficile and could improve detection. In some patients with multiple samples a low detection limit of routine culture testing at the initial or the final sampling points was noticed (Table 3). The higher sensitivity of molecular tests in these cases could therefore contribute to early detection of $C$. difficile and could have an impact on successful treatment of the disease.

\section{REFERENCES}

Alonso, R., Muñoz, C., Gros, S., García de Viedma, D., Peláez, T. \& Bouza, E. (1999). Rapid detection of toxigenic Clostridium difficile from stool samples by a nested PCR of toxin B gene. J Hosp Infect 41, 145-149.

Babady, N. E., Stiles, J., Ruggiero, P., Khosa, P., Huang, D., Shuptar, S., Kamboj, M. \& Kiehn, T. E. (2010). Evaluation of the Cepheid Xpert Clostridium difficile Epi assay for diagnosis of Clostridium difficile infection and typing of the NAP1 strain at a cancer hospital. J Clin Microbiol 48, 4519-4524.

Barbut, F., Braun, M., Burghoffer, B., Lalande, V. \& Eckert, C. (2009). Rapid detection of toxigenic strains of Clostridium difficile in diarrheal stools by real-time PCR. J Clin Microbiol 47, 1276-1277.

Bélanger, S. D., Boissinot, M., Clairoux, N., Picard, F. J. \& Bergeron, M. G. (2003). Rapid detection of Clostridium difficile in feces by realtime PCR. J Clin Microbiol 41, 730-734.

Bidet, P., Barbut, F., Lalande, V., Burghoffer, B. \& Petit, J. C. (1999). Development of a new PCR-ribotyping method for Clostridium difficile based on ribosomal RNA gene sequencing. FEMS Microbiol Lett 175, 261-266.
Braun, V., Hundsberger, T., Leukel, P., Sauerborn, M. \& Von EichelStreiber, C. (1996). Definition of the single integration site of the pathogenicity locus in Clostridium difficile. Gene 181, 29-38.

Crobach, M. J. T., Dekkers, O. M., Wilcox, M. H. \& Kuijper, E. J. (2009). European Society of Clinical Microbiology and Infectious Diseases (ESCMID): data review and recommendations for diagnosing Clostridium difficile-infection (CDI). Clin Microbiol Infect 15, 1053-1066.

Doing, K. M., Hintz, M. S., Keefe, C., Horne, S., LeVasseur, S. \& Kulikowski, M. L. (2010). Reevaluation of the Premier Clostridium difficile toxin A and B immunoassay with comparison to glutamate dehydrogenase common antigen testing evaluating Bartels cytotoxin and Prodesse ProGastro Cd polymerase chain reaction as confirmatory procedures. Diagn Microbiol Infect Dis 66, 129-134.

Eastwood, K., Else, P., Charlett, A. \& Wilcox, M. (2009). Comparison of nine commercially available Clostridium difficile toxin detection assays, a real-time PCR assay for $C$. difficile $t c d \mathrm{~B}$, and a glutamate dehydrogenase detection assay to cytotoxin testing and cytotoxigenic culture methods. J Clin Microbiol 47, 3211-3217.

Huang, H., Weintraub, A., Fang, H. \& Nord, C. E. (2009). Comparison of a commercial multiplex real-time PCR to the cell cytotoxicity neutralization assay for diagnosis of Clostridium difficile infections. J Clin Microbiol 47, 3729-3731.

Knetsch, C. W., Bakker, D., De Boer, R. F., Sanders, I., Hofs, S., Kooistra-Smid, A. M. D., Corver, J., Eastwood, K., Wilcox, M. H. \& Kuijper, E. J. (2011). Comparison of real-time PCR techniques to cytotoxigenic culture methods for diagnosing Clostridium difficile infection. J Clin Microbiol 49, 227-231.

Kvach, E. J., Ferguson, D., Riska, P. F. \& Landry, M. L. (2010). Comparison of BD GeneOhm Cdiff real-time PCR assay with a twostep algorithm and a toxin $\mathrm{A} / \mathrm{B}$ enzyme-linked immunosorbent assay for diagnosis of toxigenic Clostridium difficile infection. J Clin Microbiol 48, 109-114.

Norén, T., Alriksson, I. \& Andersson, J., Åkerlund, T. \& Unemo, M. (2011). Rapid and sensitive loop-mediated isothermal amplification test for Clostridium difficile diagnosis challenges cytotoxin B cell test and culture as gold standard. J Clin Microbiol 49, 710-711.

Novak-Weekley, S. M., Marlowe, E. M., Miller, J. M., Cumpio, J., Nomura, J. H., Vance, P. H. \& Weissfeld, A. (2010). Clostridium difficile testing in the clinical laboratory by use of multiple testing algorithms. J Clin Microbiol 48, 889-893.

Planche, T., Aghaizu, A., Holliman, R., Riley, P., Poloniecki, J., Breathnach, A. \& Krishna, S. (2008). Diagnosis of Clostridium difficile infection by toxin detection kits: a systematic review. Lancet Infect Dis 8, 777-784.

Rupnik, M. (2008). Heterogeneity of large clostridial toxins: importance of Clostridium difficile toxinotypes. FEMS Microbiol Rev 32, 541-555.

Rupnik, M., Wilcox, M. H. \& Gerding, D. N. (2009). Clostridium difficile infection: new developments in epidemiology and pathogenesis. Nat Rev Microbiol 7, 526-536.

Stamper, P. D., Babiker, W., Alcabasa, R., Aird, D., Wehrlin, J., Ikpeama, I., Gluck, L. \& Carroll, K. C. (2009). Evaluation of a new commercial TaqMan PCR assay for direct detection of the Clostridium difficile toxin B gene in clinical stool specimens. J Clin Microbiol 47, 3846-3850.

Stubbs, S., Rupnik, M., Gibert, M., Brazier, J., Duerden, B. \& Popoff, M. (2000). Production of actin-specific ADP-ribosyltransferase (binary toxin) by strains of Clostridium difficile. FEMS Microbiol Lett 186, 307-312.

Swindells, J., Brenwald, N., Reading, N. \& Oppenheim, B. (2010). Evaluation of diagnostic tests for Clostridium difficile infection. J Clin Microbiol 48, 606-608. 
Tenover, F. C., Novak-Weekley, S., Woods, C. W., Peterson, L. R., Davis, T., Schreckenberger, P., Fang, F. C., Dascal, A., Gerding, D. N. \& other authors (2010). Impact of strain type on detection of toxigenic Clostridium difficile: comparison of molecular diagnostic and enzyme immunoassay approaches. J Clin Microbiol 48, 3719-3724.

Terhes, G., Urbán, E., Sóki, J., Nacsa, E. \& Nagy, E. (2009). Comparison of a rapid molecular method, the BD GeneOhm Cdiff assay, to the most frequently used laboratory tests for detection of toxin-producing Clostridium difficile in diarrheal feces. J Clin Microbiol 47, 3478-3481.
Van den Berg, R. J., Bruijnesteijn van Coppenraet, L. S. Gerritsen, H. J., Endtz, H. P., Van der Vorm, E. R. \& Kuijper, E. J. (2005). Prospective multicenter evaluation of a new immunoassay and real-time PCR for rapid diagnosis of Clostridium difficile-associated diarrhea in hospitalized patients. J Clin Microbiol 43, 5338-5340.

Wroblewski, D., Hannett, G. E., Bopp, D. J., Dumyati, G. K., Halse, T. A., Dumas, N. B. \& Musser, K. A. (2009). Rapid molecular characterization of Clostridium difficile and assessment of populations of C. difficile in stool specimens. J Clin Microbiol 47, 2142-2148. 